\title{
Hyperactive macrophages link heart and joint disease
}

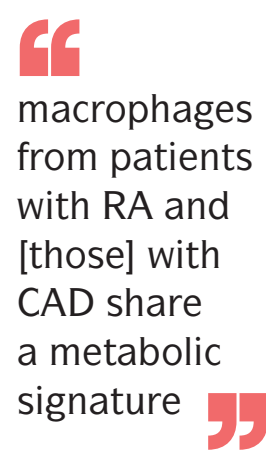

Inflammatory macrophages have a prominent role in both synovitis in rheumatoid arthritis (RA) and atherosclerotic plaques in coronary artery disease (CAD). A study now published in Annals of the Rheumatic Diseases explores whether common pathogenic mechanisms underlie the effector functions of these cells in RA and CAD.

"Patients with RA are at high risk for accelerated cardiovascular disease and currently it is unknown

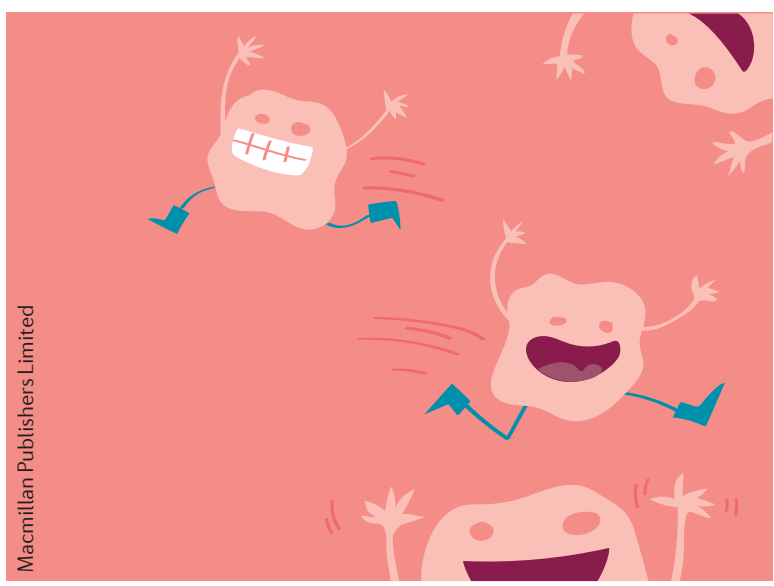

what the cellular and molecular mechanisms are that render these patients susceptible to cardiovascular complications," says corresponding author Cornelia Weyand. The findings of the new study show that macrophages from patients with RA and from patients with CAD share a metabolic signature associated with functional consequences that promote tissue-destructive inflammation.

Using macrophages generated from $\mathrm{CD} 14^{+}$precursor cells isolated from the peripheral blood of 68 patients with CAD (who had one or more myocardial infarctions), 74 patients with seropositive RA and 50 age-matched healthy controls, Weyand and colleagues found that macrophages from patients with RA or CAD consumed more oxygen and generated more ATP than macrophages from healthy controls. This hyperactivity was dependent on inactivation of the enzyme glycogen synthase kinase $3 \beta$ (GSK3 $\beta$ ), which enabled calcium to flow from the endoplasmic reticulum to the mitochondria (via mitochondriaassociated membranes) in RA and CAD macrophages.

"In macrophages from patients with RA or CAD, GSK $3 \beta$ fails to protect the mitochondria from fuel overload," explains Weyand. "As a result, mitochondria are highly active and supply the macrophages with energy and metabolic intermediates that promote inflammatory effector functions," including sustained production of the collagenase cathepsin $\mathrm{K}$.

The researchers are now exploring whether restoring the active state of GSK3 $\beta$ might curb inflammatory responses and provide a new approach to address the increased cardiovascular risk in patients with RA.

Sarah Onuora

ORIGINAL ARTICLE Zeisbrich, M. et al. Hypermetabolic macrophages in rheumatoid arthritis and coronary artery disease due to glycogen synthase kinase $3 \mathrm{~b}$ inactivation. Ann. Rheum. Dis. https://doi.org/10.1136/ annrheumdis-2017-212647 (2018) 